\title{
Development of diagnostic molecular markers for marker-assisted breeding against bacterial wilt in tomato
}

\author{
Alebel Mekuriaw Abebe ${ }^{\dagger 1)}$, Jinwoo Choi ${ }^{\dagger 1)}$, Youngjun Kim ${ }^{1)}$, Chang-Sik Oh$^{2)}$, Inhwa Yeam ${ }^{3)}$, Ill-Sup Nou ${ }^{4)}$ \\ and Je Min Lee*1) \\ 1) Department of Horticultural Science, Kyungpook National University, Daegu 41566, South Korea \\ 2) Department of Horticultural Biotechnology, College of Life Science, Kyung Hee University, Yongin, Gyeonggi-do 17104, South \\ Korea \\ 3) Department of Horticulture and Breeding, Andong National University, Andong, Gyeongbuk, 36729, South Korea \\ 4) Department of Horticulture, Sunchon National University, Suncheon, Jeonnam 57922, South Korea
}

\begin{abstract}
Bacterial wilt, caused by the Ralstonia pseudosolanacearum species complex, is an important vascular disease that limits tomato production in tropical and subtropical regions. Two major quantitative trait loci (QTL) of bacterial wilt resistance on chromosome $6(\mathrm{Bwr}-6)$ and $12(\mathrm{Bwr}-12)$ were previously identified in Solanum lycopersicum 'Hawaii 7996'; however, marker-assisted breeding for bacterial wilt resistance is not well established. To dissect the QTL, six cleaved amplified polymorphic sites (CAPS) and derived CAPS (dCAPS) markers within the Bwr-6 region and one dCAPS marker near Bwr-12 were developed, and resistance levels in 117 tomato cultivars were evaluated. Two markers, RsR6-5 on chromosome 6 and RsR12-1 on chromosome 12, were selected based on the genotypic and phenotypic analysis. The combination of RsR6-5 and RsR12-1 effectively distinguishes resistant and susceptible cultivars. Furthermore, the efficiency of the two markers was validated in the $F_{3}$ generation derived from the $F_{2}$ population between E6203 (susceptible) and Hawaii 7998 (resistant). Resistant alleles at both loci led to the resistance to bacterial wilt. These markers will facilitate marker-assisted breeding of tomato resistant to bacterial wilt.
\end{abstract}

Key Words: tomato, bacterial wilt, polygenic resistance, molecular marker, single nucleotide polymorphism, marker-assisted breeding.

\section{Introduction}

Bacterial wilt, caused by Ralstonia solanacearum, is one of the most destructive diseases that affects many plant species. The $R$. solanacearum species complex (RSSC) has been classified into races, biovars, and phylotypes based on host range, carbon source usage, 16S-23S rRNA gene sequence of the strains, respectively (Cho et al. 2018, Hayward 1991, Jeong et al. 2007). Genomic analysis and proteomic profiling of various strains of the pathogen collected from different countries classified RSSC into three species: $R$. solanacearum (Phylotype II), R. pseudosolanacearum (Phylotypes I and III), and R. syzygii (Phylotype IV). Further genomic analysis of the species classified R.syzygii into three subspecies named syzygii, indonesiensis, and celebesensis (Prior et al. 2016).

\section{Communicated by Sang-Nag Ahn}

Received March 10, 2020. Accepted June 3, 2020.

First Published Online in J-STAGE on August 4, 2020.

*Corresponding author (e-mail: jemin@knu.ac.kr)

${ }^{\dagger}$ These authors contributed equally to this work
The pathogen has a wide host range. Tomato and other Solanaceae plants are major hosts. The disease threatens the cultivation of these crops in tropical and subtropical regions and heated greenhouses in temperate regions because high temperatures are better suited for the pathogen and disease development. As a result, the pathogen causes substantial economic losses (Hayward 1991, Lopes and Rossato 2018). The pathogen moves into plant roots via natural openings, such as hydathodes, or damaged areas and proliferates in the xylem tissues. It then damages the xylem tissues and blocks the water flow, leading to the total collapse and death of susceptible plants (Bae et al. 2015, Lowe-Power et al. 2018). Xylem colonization and spread are necessary for bacterial wilt disease progress because mutations in xylem colonization rendered pathogen strains incapable of causing wilting in tomato plants (Schell 2000). Evaluation of core collections of the three fruit vegetables of Solanaceae crops (tomato, eggplant, and pepper) against different strains of the pathogen showed that resistance of tomato collections is low compared with eggplant and pepper (Lebeau et al. 2011).

Different control strategies, such as chemical, biological, 
and cultural practices, can reduce bacterial wilt severity, but none of them are effective (Yuliar et al. 2015). Development and use of resistant cultivars is the most effective approach to control bacterial wilt (Abebe et al. 2016, Huet 2014, Scott et al. 2005, Wang et al. 2018). Breeding for bacterial wilt-resistant cultivars has been challenging due to the polygenic nature of resistance, broad host range, variability of the pathogen strains, and effect of environmental factors that directly influence the phenotypic expression of the disease (Danesh et al. 1994, Fegan and Prior 2005, Hayward 1991, Lee et al. 2011, Thoquet et al. 1996b, Tran and Kim 2010). Moreover, the resistance locus is linked to undesirable horticultural traits (Scott et al. 2005). Solanum lycopersicum 'Hawaii 7996' (hereafter Hawaii 7996) is a stable resistant resource against bacterial wilt across various geographic locations and different bacterial strains with the highest average survival rate of $97 \%$ (Wang et al. 1998). Analysis of bacterial wilt resistance using $F_{2}, F_{3}$, and recombinant inbred line (RIL) populations derived from a cross between Hawaii 7996 (resistant) and West Virginia 700 (susceptible) identified QTL on chromosomes 4, 6, and 11 (Thoquet et al. 1996a); 3, 4, 6, 8, and 10 (Thoquet et al. 1996b); 6 (Mangin et al. 1999); 6, 8, and 12 (Wang et al. 2000); 3, 4, and 6 (Carmeille et al. 2006); and 3, 6, and 12 (Wang et al. 2013). Among them, a QTL on chromosome 6 (Bwr-6) was stable when measured with different phenotyping criteria (area under disease progress curve and bacterial colonization), in different bacterial strains (race 3phylotype II and race 1-phylotype I), and as measured in two different seasons (hot and cold) (Carmeille et al. 2006). Bwr-12 was an active QTL specifically against Pss4 (race 1, biovar 4) (Wang et al. 2000). QTL on chromosome 6 (Bwr-6) and 12 (Bwr-12) are thought to be responsible for the stable resistance of Hawaii 7996. Bwr-12 covers $2.8 \mathrm{cM}$ between the SSR markers SLM12-12 and SLM12-2, controlling more than $50 \%$ of the phenotypic variation in some trials. Bwr-6 was localized between SLM6-124 and SLM6-110 covering $15.5 \mathrm{cM}$ and controlling up to $22.2 \%$ of the phenotypic variation (Wang et al. 2013). Although two QTL, Bwr-6 and Bwr-12, were repeatedly confirmed as major contributors to bacterial wilt resistance in Hawaii 7996, the genetic nature of these critical QTL remained unidentified. We have previously conducted whole-genome resequencing of two susceptible cultivars (Heinz 1706 and BWS-3) and seven resistant cultivars (Hawaii 7996, Hawaii 7998, 10-BA-3-33, 10-BA-4-24, BWR-1, BWR-22, and BWR-23) of tomato and identified genome-wide single nucleotide polymorphisms (SNPs) in resistant and susceptible groups of cultivars (Kim et al. 2018). The highest number of polymorphic SNPs in coding regions were found on chromosome 12 (168 SNPs) followed by chromosome 6 (53 SNPs). These SNPs might be associated with resistance to bacterial wilt. Based on the SNP information generated by re-sequencing, an HRM marker (KHU-1) that is tightly linked to $B w r-12$ was developed; however, no tightly linked SNP-based molecular marker was developed to trace Bwr-6 resistance due to the large interval ( 12.7 Mbp) (Kim et al. 2018).

In this study, we further analyzed the Bwr-6 region to dissect and develop a diagnostic molecular marker for this important QTL. Cleaved amplified polymorphic site (CAPS) and derived CAPS (dCAPS) markers were developed within Bwr-6 and screened for their diagnostic potential using 117 tomato genotypes. Phenotypic and genotypic analysis using a wide range of germplasms enabled us to select RsR6-5 as a diagnostic marker for Bwr-6 among the newly developed markers in the region. Consequently, this marker, in combination with RsR12-1, effectively distinguished bacterial wilt-resistant and wilt-susceptible tomato cultivars. The newly developed marker RsR6-5 together with RsR12-1 will promote marker-assisted breeding of tomato by targeting two major resistance QTL against bacterial wilt.

\section{Materials and Methods}

\section{Plant materials}

In total, 117 tomato cultivars were collected (either seed or genomic DNA sample) from different sources, including the Tomato Genetics Resource Center (TGRC), UC Davis; Kyung Hee University, Korea; National Agrobiodiversity Center (RDA-Genebank), Korea; and various commercial seed companies in Korea. The phenotype of 27 cultivars was confirmed by inoculation test in this study, 12 cultivars were inferred from previous reports and the phenotype of 78 cultivars was received from the respective company/ supplier along with the genomic DNA sample (Table 1). Seeds were first sown in Petri dishes for germination, and germinated seeds were transferred to 128 cell seedling trays "( $28 \times 28 \times 40$, bottom $15 \mathrm{~mm})$ " filled with bio mix (JM bio, Korea). The seedlings were grown in the Agricultural Experiment Station of Kyungpook National University in a glasshouse at an average temperature of $25-28^{\circ} \mathrm{C}$ and $16-$ $8 \mathrm{~h}$ light-dark cycles. The seedlings were moved from the glasshouse to the growth chamber 3-4 days before inoculation for acclimatization to growth chamber conditions where they were kept post-inoculation. Four-week-old seedlings were used for inoculation.

Table 1. Summary of the phenotypic composition of tomato cultivars used in this study

\begin{tabular}{lccc}
\hline \hline \multirow{2}{*}{ Phenotyping } & \multirow{2}{*}{$\begin{array}{c}\text { Number of } \\
\text { cultivars }\end{array}$} & \multicolumn{2}{c}{ Bacterial wilt phenotype } \\
\cline { 3 - 4 } & 27 & Resistant & Susceptible \\
\hline This study & 12 & 11 & 16 \\
Previous report & 78 & 2 & 8 \\
Company/supplier & 117 & 17 & 100 \\
\hline Total & &
\end{tabular}


Disease evaluation of tomato cultivars for bacterial wilt resistance

R. pseudosolanacearum strain SL882, classified as race 1, biovar 4, and phylotype I (Lee et al. 2011), was cultured on casamino acid-peptone-glucose (CPG) medium (casamino acid, $1 \mathrm{~g}$; peptone, $10 \mathrm{~g}$; glucose, $5 \mathrm{~g}$; and agar, $15 \mathrm{~g}$ per liter of distilled water) and incubated at $28^{\circ} \mathrm{C}$ for $48 \mathrm{~h}$ (Kelman 1954). The bacterial culture from the Petri dish $(90 \times 15 \mathrm{~mm})$ was rinsed with distilled water and washed using a cotton swab to make the inoculum suspension and its concentration was adjusted to approximately $10^{8} \mathrm{CFU} / \mathrm{ml}\left(\mathrm{OD}_{600} \sim 0.1\right)$ using a NanoDrop 2000/UV-Vis spectrophotometer (ThermoFisher Scientific, Waltham, MA, USA).

Seedlings at one month after sowing were inoculated by dipping the roots in the bacterial suspension (Caldwell et al. 2017). Each seedling was grown in a 128-cell seedling tray $(28 \times 28 \times 40$, bottom $15 \mathrm{~mm})$ was pulled out, and its roots were dipped in the bacterial suspension. The inoculated seedlings were transplanted into 50 -cell seedling trays $(45 \times 45 \times 50$, bottom $32 \mathrm{~mm})$ and kept in a growth chamber (temperature $=28^{\circ} \mathrm{C}$, relative humidity $=70 \%$, and 16 $8 \mathrm{~h}$ light-dark cycles). The disease severity was evaluated based on a disease scale of 0 to 4 , where $0=$ no visible symptoms; $1=25 \%$ of leaves wilting; $2=50 \%$ of leaves wilting; $3=75 \%$ leaves wilting; $4=$ all foliage is wilted, and the plant dies (Morel et al. 2018). The disease scale was determined based on visual observation of the degree of wilting. The average value of disease severity for ten plants was calculated per each line. Cultivars with mean disease severity scores of $<2$ were classified as resistant, while those with scores $>2$ were classified as susceptible to bacterial wilt.

\section{Genomic DNA extraction}

Genomic DNA of 27 cultivars which were phenotyped in this study was extracted from young leaf tissues with a modified cetyltrimethylammonium bromide (CTAB) method (Murray and Thompson 1980). The concentration and quality of genomic DNA were measured using NanoDrop 2000/UV-Vis spectrophotometer (ThermoFisher Scientific, Waltham, MA, USA).

\section{Sequence analysis of Bwr-6 region and marker develop- ment}

Non-synonymous SNPs between resistant and susceptible groups of tomato cultivars near Bwr-6 within 15 candidate genes (18 SNPs) were previously identified by wholegenome resequencing (Kim et al. 2018). Each candidate gene contained one non-synonymous SNP except two genes, Solyc06g051110.1 and Solyc06g051140.2, which have two and three SNPs, respectively. The nucleotide changes between bacterial wilt-resistant and wiltsusceptible groups of tomato varieties for these SNPs, along with the respective amino acid changes, are presented in Table 2. To dissect Bwr-6 and develop diagnostic markers, selected SNPs were converted to CAPS/dCAPS markers. Based on six non-synonymous SNPs located at 24669159, 34389374, 34399541, 35950028, 37049726, and 37186202, respectively) in Bwr-6 (Kim et al. 2018), five CAPS (RsR6-1 RsR6-5) and one dCAPS (RsR6-6) markers were developed. An HRM molecular marker (KHU-1),

Table 2. List of candidate genes containing non-synonymous SNPs near Bwr-6 and Bwr-12, adapted from Kim et al. (2018). SNPs indicated in bold were used to develop CAPS/dCAPS markers in this study

\begin{tabular}{|c|c|c|c|c|c|c|}
\hline \multirow{2}{*}{ Candidate gene } & \multirow{2}{*}{$\begin{array}{l}\text { SNP position } \\
\text { (bp) }\end{array}$} & \multicolumn{2}{|c|}{ Nucleotide change } & \multicolumn{2}{|c|}{ Amino acid change } & \multirow{2}{*}{ Gene annotation (ITAG2.4) } \\
\hline & & Susceptible & Resistant & Susceptible & Resistant & \\
\hline Solyc06g035530.2 & $24,482,686$ & $\mathrm{~T}$ & $\mathrm{C}$ & $\mathrm{F}$ & $\mathrm{L}$ & Gibberellin 20-oxidase-2 \\
\hline Solyc06g035620.2 & $24,667,815$ & A & G & Y & $\mathrm{C}$ & Scarecrow-like 1 transcription factor \\
\hline Solyc06g035630.1 & $24,669,159$ & $\mathbf{T}$ & $\mathbf{C}$ & $\mathbf{L}$ & $\mathbf{P}$ & GRAS family transcription factor \\
\hline Solyc06g036060.2 & $25,438,944$ & A & G & I & V & Zinc finger family protein \\
\hline Solyc06g048580.1 & $31,287,788$ & $\mathrm{~T}$ & $\mathrm{C}$ & $\mathrm{L}$ & S & Unknown protein \\
\hline Solyc06g051110.1 & $34,213,688$ & G & $\mathrm{T}$ & $\mathrm{L}$ & $\mathrm{F}$ & Unknown protein \\
\hline Solyc06g051110.1 & $34,214,085$ & $\mathrm{C}$ & $\mathrm{T}$ & A & $\mathrm{V}$ & Unknown protein \\
\hline Solyc06g051140.2 & $34,285,452$ & A & $\mathrm{C}$ & $\mathrm{E}$ & A & Ubiquitin-conjugating enzyme 22 \\
\hline Solyc06g051140.2 & $34,285,469$ & G & A & M & I & Ubiquitin-conjugating enzyme 22 \\
\hline Solyc06g051140.2 & $34,285,734$ & $\mathrm{C}$ & A & A & $\mathrm{D}$ & Ubiquitin-conjugating enzyme 22 \\
\hline Solyc06g051150.1 & $34,291,655$ & $\mathrm{C}$ & A & $\mathrm{L}$ & I & Pentatricopeptide repeat-containing protein \\
\hline Solyc06g051190.1 & $34,389,374$ & $\mathbf{A}$ & $\mathbf{G}$ & $\mathbf{T}$ & $\mathbf{A}$ & RNA-dependent RNA polymerase family protein \\
\hline Solyc06g051210.2 & $34,399,541$ & $\mathbf{C}$ & $\mathbf{T}$ & $\mathbf{P}$ & $\mathbf{S}$ & Bromodomain-containing protein \\
\hline Solyc06g053210.2 & $35,950,028$ & $\mathbf{T}$ & $\mathbf{C}$ & $\mathbf{X}$ & $\mathbf{Q}$ & Ubiquitin | Homeobox leucine zipper protein \\
\hline Solyc06g054000.1 & $36,868,039$ & G & A & $\mathrm{V}$ & I & Unknown protein \\
\hline Solyc06g054200.1 & $37,021,520$ & A & $\mathrm{G}$ & $\mathrm{R}$ & $\mathrm{S}$ & Calmodulin protein kinase \\
\hline Solyc06g054230.2 & $37,049,726$ & $\mathbf{T}$ & $\mathbf{G}$ & D & $\mathbf{E}$ & Calmodulin protein kinase \\
\hline Solyc06g054400.2 & $37,186,202$ & $\mathbf{T}$ & $\mathbf{C}$ & I & $\mathbf{T}$ & Translation initiation factor \\
\hline Solyc12g009690.1 & $2,941,301$ & $\mathbf{A}$ & $\mathbf{G}$ & $\mathbf{H}$ & $\mathbf{R}$ & LRR receptor-like serine/threonine-protein kinase \\
\hline
\end{tabular}


Table 3. DNA marker information used in this study

\begin{tabular}{|c|c|c|c|c|c|c|c|}
\hline \multirow{2}{*}{$\begin{array}{l}\text { Marker } \\
\text { name }\end{array}$} & \multirow{2}{*}{$\begin{array}{l}\text { SNP position } \\
\text { (bp) }\end{array}$} & \multirow{2}{*}{$\begin{array}{l}\text { Marker } \\
\text { type }\end{array}$} & \multirow{2}{*}{ Primer sequence $\left(5^{\prime} \rightarrow 3^{\prime}\right)$} & \multirow{2}{*}{$\begin{array}{c}\mathrm{T}_{\mathrm{m}} \\
\left({ }^{\circ} \mathrm{C}\right)\end{array}$} & \multirow{2}{*}{$\begin{array}{c}\text { Restriction } \\
\text { enzyme }\end{array}$} & \multicolumn{2}{|c|}{ Expected size (bp) } \\
\hline & & & & & & Susceptible & Resistant \\
\hline \multirow[t]{2}{*}{ RsR6-1 } & $24,669,159$ & CAPS & F: GGAAATATTGGTTACAATCCAGTG & 57.5 & MnlI & 227 & 173,54 \\
\hline & & & R: GAATACAACAAATCACTACCGGTC & 59.3 & & & \\
\hline \multirow[t]{2}{*}{ RsR6-2 } & $34,389,374$ & CAPS & F: CTTCTTGATAGGACGACGTGATAT & 59.3 & RsaI & 87,116 & 203 \\
\hline & & & R: CAATCAACGGATCACCCATTTTTC & 59.3 & & & \\
\hline \multirow[t]{2}{*}{ RsR6-3 } & $34,399,541$ & CAPS & F: CTCTTTTTGCCAGATCTTGAATAG & 57.5 & $M n l \mathrm{I}$ & 214 & 116,98 \\
\hline & & & R: CCATAGGTCAGCATCAAATTTCAA & 57.5 & & & \\
\hline \multirow[t]{2}{*}{ RsR6-4 } & $35,950,028$ & CAPS & F: GTTTTCCTTGCAAATCATTTTGGC & 57.5 & MseI & 116,97 & 213 \\
\hline & & & R: GTATATGTTGAGTTCACAATTCCC & 57.5 & & & \\
\hline \multirow[t]{2}{*}{ RsR6-5 } & $37,049,726$ & CAPS & F: CTCAGAAACTGGATAAACTCGAAG & 59.3 & Hinf $\mathrm{I}$ & 204 & 129,75 \\
\hline & & & R: GGAGAAAGCAGCCAGCCATTTTT & 60.6 & & & \\
\hline \multirow[t]{2}{*}{ RsR6-6 } & $37,186,202$ & dCAPS & F: CGGTGATGAGCAGGATTGATAAAA & 59.3 & НруСH4III & 234 & 200,34 \\
\hline & & & R: AGTCTTGGCCTTTGACGTGAAAGTGACACAAGAAG & 60.6 & & & \\
\hline \multirow[t]{2}{*}{ RsR12-1 } & $2,941,301$ & dCAPS & F: GTTACACGAACAAGCTTAAATTTCTAGATTTATCCC & 58.8 & AciI & 203 & 168,35 \\
\hline & & & $\mathrm{R}$ GTAATCAATTCGAAGGACCTGTC & 64.9 & & & \\
\hline
\end{tabular}

tightly linked to bacterial wilt resistance on chromosome 12, was developed based on an $\operatorname{SNP}(\mathrm{A} / \mathrm{G})$ located at 2,941,301 bp (Kim et al. 2018). We converted this HRM marker, KHU-1, to a dCAPS marker (RsR12-1) to trace Bwr-12 in this study (Table 3). The sequence of the target genes was retrieved from the Sol Genomics Network (https://solgenomics.net/). dCAPS finder 2.0 (http://helix. wustl.edu/dcaps/dcaps.html) NEBcutter (http://nc2.neb.com/ NEBcutter2/) were used to find the appropriate restriction enzymes for the respective SNP site.

\section{PCR amplification and gel electrophoresis}

PCR reactions were carried out according to the manufacturer's instructions (SolGent Co., Ltd., Daejeon, Korea) in a total volume of $25 \mu \mathrm{l}$ containing $1 \mu \mathrm{l}$ genomic DNA, $2.5 \mu \mathrm{l} 10 \mathrm{X} e$-Tag reaction buffer, $0.5 \mu \mathrm{l}$ of $10 \mathrm{mM} \mathrm{dNTP}$ mix, $1 \mu \mathrm{l}$ of each forward and reverse primers, $0.125 \mu \mathrm{l}$ Solg e-Taq DNA polymerase, and $18.875 \mu \mathrm{l}$ of $\mathrm{ddH}_{2} \mathrm{O}$. PCR amplification was carried out using a Bio-Rad T100 thermocycler (Bio-Rad Laboratories, Inc.) with the following conditions: denaturing for $3 \mathrm{~min}$ at $95^{\circ} \mathrm{C}$, followed by 34 cycles of $30 \mathrm{~s}$ at $95^{\circ} \mathrm{C}$ denaturation, $30 \mathrm{~s}$ at annealing temperature (which varied for different primer sets (Table 3)), $1 \mathrm{~min}$ at $72^{\circ} \mathrm{C}$ extension, and a final elongation step at $72^{\circ} \mathrm{C}$ for $5 \mathrm{~min}$. PCR products were digested with the respective restriction enzymes. The reaction mixture consisted of $5 \mu \mathrm{l}$ template PCR product, $1 \mu \mathrm{l}$ reaction buffer, $0.1 \mu \mathrm{l}$ of restriction enzyme, and $3.9 \mu \mathrm{l} \mathrm{ddH}_{2} \mathrm{O}$. The mixture was incubated at $37^{\circ} \mathrm{C}$ for $16 \mathrm{hrs}$ and was carried out using Bio-Rad T100 thermal cycler (Bio-Rad Laboratories, Inc.). The digested product was mixed with $2 \mu \mathrm{l} 6 \mathrm{X}$ DNA loading buffer and subjected to gel electrophoresis on a $3 \%$ agarose gel to visualize the polymorphic DNA bands. The details of CAPS/dCAPS marker information, including primer sequences, restriction enzymes, and the expected band sizes for resistant and susceptible tomato groups, are presented in Table 3 .

\section{Selection of $F_{3}$ and marker validation}

To confirm the efficiency of the two QTLs, $\mathrm{F}_{2}$ generation developed from a cross between E6203 (susceptible) and Hawaii 7998 (resistant) were screened with two markers RsR6-5 (Bwr-6) and RsR12-1 (Bwr-12). Ten $\mathrm{F}_{2}$ plants homozygous for both markers (five resistant and five susceptible), four $\mathrm{F}_{2}$ plants harboring only RsR6-5, and four $\mathrm{F}_{2}$ plants harboring only RsR12-1 were selected for harvesting the $\mathrm{F}_{3}$ seeds. The $\mathrm{F}_{3}$ generation were inoculated and evaluated for disease resistance. Ten plants of each $\mathrm{F}_{3}$ progeny were inoculated. The resistant and susceptible parents were included as controls during disease evaluation. The mean disease severity of ten plants was used to designate the resistance level of each progeny. Furthermore, the markers were evaluated for their diagnostic value using tomato cultivars (Yang et al. 2015).

\section{Results}

Phenotyping of tomato cultivars for bacterial wilt resistance

The resistance level of 27 cultivars against bacterial wilt disease was inoculated and confirmed in this study. Hawaii 7996, B-Blocking, Shincheonggang, BWR-20, Spider, High Power, 10-BA-3-33, 10-BA-4-24, IT 201664, Hawaii 7998, and Fighting were resistant with low mean disease severity scores $(<2)$ while UC-134, LA1589, Purple Calabash, Florida8516, Heinz 1706, A-1, E6203, Moneymaker, Super Dotaerang, Anahu, Red Strong, Bluck Plum, Gold Nugget, VF-36, M82, and Dotaerang Red were susceptible with a mean disease severity score of $>2.0$ (Fig. 1). Previously reported susceptible cultivars, such as Moneymaker, Heinz 1706, and Super Dotaerang (Han et al. 2009, Kim et al. 2018), showed the highest mean disease severity indicating the presence of adequate disease pressure on inoculated plants. In addition, phenotypic information of 12 cultivars was inferred from previous reports and 78 cultivars were received from respective companies. In total, the 


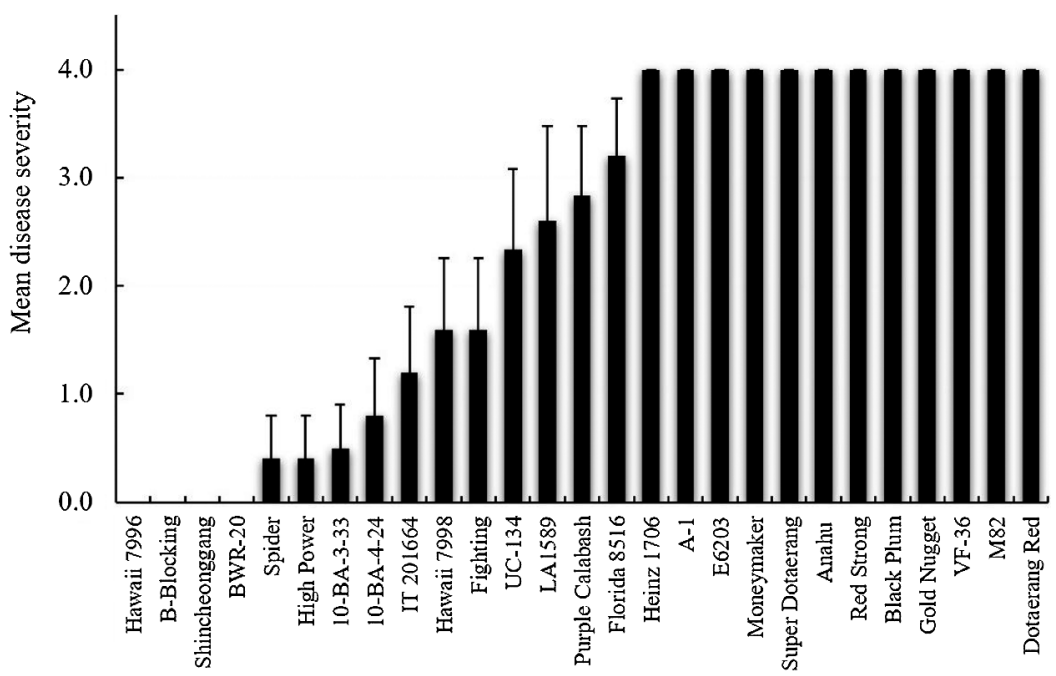

Fig. 1. Mean disease severity of tomato cultivars infected with $R$. pseudosolanacearum strain SL882, one month after inoculation. The disease severity was scored based on the disease scale of 0 to 4 , where $0=$ no visible symptoms; $1=25 \%$ of leaves wilting; $2=50 \%$ of leaves wilting; $3=75 \%$ of leaves wilting; and $4=$ all foliage is wilted, and the plant dies.

collection includes 17 resistant and 100 susceptible cultivars (Table 1).

\section{Screening of the SNP markers using phenotypic data of tomato cultivars}

The primer sets were tested for polymorphism using six susceptible and five resistant cultivars. All primer sets resulted in a clear polymorphic band between susceptible and resistant tomato cultivars after enzyme digestion (Fig. 2). For selecting an accurate and reliable marker for Bwr-6, six markers (RsR6-1 6-6) were screened using 117 tomato cultivars (Table 4). The Bwr-12 genotype of these cultivars was determined using RsR12-1. Hawaii 7996, Hawaii 7998, 10-BA-3-33, 10-BA-4-24, BWR-20, BWR-1, BWR-22, and BWR-23 had homozygous resistant genotypes with all six markers near Bwr-6. IT201664, High Power, Spider, SVTX6258, Super High Power, B-Blocking, Shincheonggang, Fighting, and Geumgang are either susceptible or heterozygous to RsR6-1, RsR6-2, and RsR6-3 while they are resistant to RsR6-4, RsR6-5, and RsR6-6 except for High Power, which is heterozygous to RsR6-4 and RsR6-5. Comparing the six markers based on the genotype of resistant cultivars, RsR6-1, RsR6-2, and RsR6-3 did not seem better candidate markers for Bwr-6 because IT 201664, High Power, Spider, and Super High Power are susceptible to these markers.

Therefore, we considered RsR6-4, RsR6-5, and RsR6-6 for further analysis using the susceptible set of cultivars. Almost all bacterial wilt-susceptible cultivars had susceptible or heterozygous genotypes to RsR6-5 except Gold Sugar, Sinheukjinju, SV7160TC, and LA1589. Red Strong shows the resistant genotype to RsR6-4 and the susceptible genotype to RsR6-5. Cultivars resistant to RsR6-6 and heterozygous with RsR12-1, such as SV02444 TG, SV4224 $\mathrm{TH}$, and SV0339TG, are expected to be resistant to bacte- rial wilt, but all were susceptible. In addition, Red Strong and SkyBall have homozygous resistant genotypes with RsR6-6 and RsR12-1, although both exhibit susceptible phenotype (Table 4).

The presence of resistant alleles in both Bwr-6 and Bwr-12 resulted in resistant phenotype while the absence of either of the two resulted in susceptible phenotype. This marker analysis indicated that cultivars homozygous resistant to RsR6-5 and either a homozygous resistant

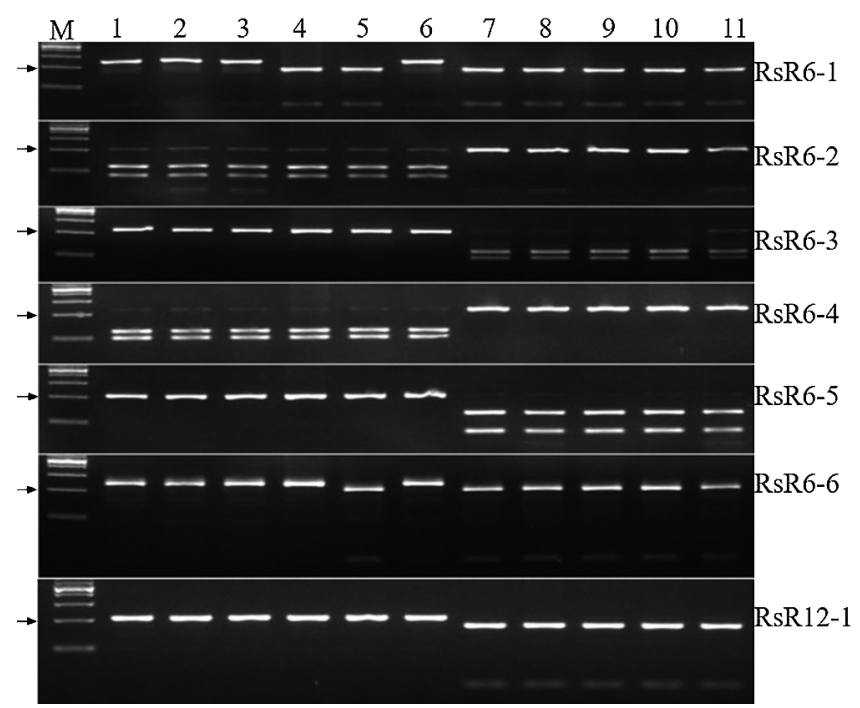

Fig. 2. Marker analysis of the newly developed CAPS/dCAPS in 11 tomato genotypes near Bwr-6 (RsR6-1 RsR6-6) and Bwr-12 (RsR12-1). Lanes 1 to 6 represent the bacterial wilt-susceptible group (M82, E6203, UC-134, VF-36, Gold Nugget, and Moneymaker) and lanes 7 to 11 represent the bacterial wilt-resistant group (Hawaii 7996, Hawaii 7998, 10-BA-3-33, 10-BA-4-24, and BWR-20). M, 1 kb DNA size marker. The arrows on the left indicate a $200 \mathrm{bp}$ standard fragment. 
Table 4. SNP marker genotype of resistant and susceptible tomato cultivars or lines used in this study

\begin{tabular}{|c|c|c|c|c|c|c|c|c|c|c|c|}
\hline \multirow{2}{*}{ No. } & \multirow{2}{*}{ Tomato cultivar/line } & \multirow{2}{*}{ Type } & \multirow{2}{*}{ Company/Supplier ${ }^{a}$} & \multicolumn{7}{|c|}{ SNP marker genotype ${ }^{b}$} & \multirow{2}{*}{ Reference $^{c}$} \\
\hline & & & & RsR6-1 & RsR6-2 & RsR6-3 & RsR6-4 & RsR6-5 & RsR6-6 & RsR12-1 & \\
\hline \multicolumn{12}{|c|}{ Bacterial wilt resistant } \\
\hline 1 & Hawaii 7996 & Inbred line & KHU & $\mathrm{R}$ & $\mathrm{R}$ & $\mathrm{R}$ & $\mathrm{R}$ & $\mathrm{R}$ & $\mathrm{R}$ & $\mathrm{R}$ & This study \\
\hline 2 & Hawaii 7998 & Inbred line & KHU & $\mathrm{R}$ & $\mathrm{R}$ & $\mathrm{R}$ & $\mathrm{R}$ & $\mathrm{R}$ & $\mathrm{R}$ & $\mathrm{R}$ & This study \\
\hline 3 & 10-BA-3-33 & Inbred line & KHU & $\mathrm{R}$ & $\mathrm{R}$ & $\mathrm{R}$ & $\mathrm{R}$ & $\mathrm{R}$ & $\mathrm{R}$ & $\mathrm{R}$ & This study \\
\hline 4 & 10-BA-4-24 & Inbred line & KHU & $\mathrm{R}$ & $\mathrm{R}$ & $\mathrm{R}$ & $\mathrm{R}$ & $\mathrm{R}$ & $\mathrm{R}$ & $\mathrm{R}$ & This study \\
\hline 5 & BWR-20 & Inbred line & KHU & $\mathrm{R}$ & $\mathrm{R}$ & $\mathrm{R}$ & $\mathrm{R}$ & $\mathrm{R}$ & $\mathrm{R}$ & $\mathrm{R}$ & This study \\
\hline 6 & BWR-1 & Inbred line & KHU & $\mathrm{R}$ & $\mathrm{R}$ & $\mathrm{R}$ & $\mathrm{R}$ & $\mathrm{R}$ & $\mathrm{R}$ & $\mathrm{R}$ & Kim et al. (2018) \\
\hline 7 & BWR-22 & Inbred line & KHU & $\mathrm{R}$ & $\mathrm{R}$ & $\mathrm{R}$ & $\mathrm{R}$ & $\mathrm{R}$ & $\mathrm{R}$ & $\mathrm{R}$ & Kim et al. (2018) \\
\hline 8 & BWR-23 & Inbred line & KHU & $\mathrm{R}$ & $\mathrm{R}$ & $\mathrm{R}$ & $\mathrm{R}$ & $\mathrm{R}$ & $\mathrm{R}$ & $\mathrm{R}$ & Kim et al. (2018) \\
\hline 9 & IT 201664 & Inbred line & $\mathrm{RDA}$ & $\mathrm{H}$ & S & S & $\mathrm{R}$ & $\mathrm{R}$ & $\mathrm{R}$ & $\mathrm{R}$ & This study \\
\hline 10 & High Power & $\mathrm{F}_{1}$ hybrid & Dae Yeon seed co. & $\mathrm{H}$ & S & S & $\mathrm{H}$ & $\mathrm{H}$ & $\mathrm{R}$ & $\mathrm{R}$ & This study \\
\hline 11 & Super High Power & $\mathrm{F}_{1}$ hybrid & Dae Yeon seed co. & S & S & S & $\mathrm{R}$ & $\mathrm{R}$ & $\mathrm{R}$ & $\mathrm{R}$ & Kim et al. (2018) \\
\hline 12 & Spider & $\mathrm{F}_{1}$ hybrid & Takii Korea & S & S & $\mathrm{S}$ & $\mathrm{R}$ & $\mathrm{R}$ & $\mathrm{R}$ & $\mathrm{R}$ & This study \\
\hline 13 & B-Blocking & $\mathrm{F}_{1}$ hybrid & Takii Korea & $\mathrm{H}$ & $\mathrm{H}$ & $\mathrm{H}$ & $\mathrm{R}$ & $\mathrm{R}$ & $\mathrm{R}$ & $\mathrm{H}$ & This study \\
\hline 14 & Shincheonggang & $\mathrm{F}_{1}$ hybrid & Farm Hannong & $\mathrm{H}$ & $\mathrm{H}$ & $\mathrm{H}$ & $\mathrm{R}$ & $\mathrm{R}$ & $\mathrm{R}$ & $\mathrm{H}$ & This study \\
\hline 15 & Fighting & $\mathrm{F}_{1}$ hybrid & Takii Korea & $\mathrm{H}$ & $\mathrm{H}$ & $\mathrm{H}$ & $\mathrm{R}$ & $\mathrm{R}$ & $\mathrm{R}$ & $\mathrm{H}$ & This study \\
\hline 16 & SVTX6258 & $\mathrm{F}_{1}$ hybrid & Monsanto Korea & $\mathrm{H}$ & $\mathrm{H}$ & $\mathrm{H}$ & $\mathrm{R}$ & $\mathrm{R}$ & $\mathrm{R}$ & $\mathrm{R}$ & Supplier \\
\hline 17 & Geumgang & $F_{1}$ hybrid & Monsanto Korea & $\mathrm{H}$ & $\mathrm{H}$ & $\mathrm{H}$ & $\mathrm{R}$ & $\mathrm{R}$ & $\mathrm{R}$ & $\mathrm{H}$ & Supplier \\
\hline \multicolumn{12}{|c|}{ Bacterial wilt susceptible } \\
\hline 18 & M82 & Inbred line & TGRC & $\mathrm{S}$ & $\mathrm{S}$ & $\mathrm{S}$ & $\mathrm{S}$ & $\mathrm{S}$ & $\mathrm{S}$ & $\mathrm{S}$ & This study \\
\hline 19 & E6203 & Inbred line & TGRC & S & S & S & S & S & S & S & This study \\
\hline 20 & VF36 & Inbred line & TGRC & $\mathrm{R}$ & S & S & S & S & S & $\mathrm{S}$ & This study \\
\hline 21 & Moneymaker & Inbred line & TGRC & $\mathrm{R}$ & S & S & S & S & S & S & This study \\
\hline 22 & A-1 & Inbred line & TGRC & S & S & S & S & $\mathrm{S}$ & S & S & This study \\
\hline 23 & Anahu & Inbred line & TGRC & S & S & $\mathrm{S}$ & $\mathrm{S}$ & S & $\mathrm{R}$ & $\mathrm{S}$ & This study \\
\hline 24 & Black Plum & Inbred line & TGRC & S & S & $\mathrm{S}$ & $\mathrm{R}$ & $\mathrm{S}$ & $\mathrm{R}$ & $\mathrm{S}$ & This study \\
\hline 25 & Florida 8516 & Inbred line & TGRC & S & S & S & S & S & S & S & This study \\
\hline 26 & Gold Nugget & Inbred line & TGRC & $\mathrm{R}$ & S & S & S & S & $\mathrm{R}$ & S & This study \\
\hline 27 & Purple Calabash & Inbred line & TGRC & $\mathrm{H}$ & $\mathrm{H}$ & $\mathrm{S}$ & $\mathrm{H}$ & $\mathrm{S}$ & $\mathrm{R}$ & $\mathrm{S}$ & This study \\
\hline 28 & UC-134 & Inbred line & TGRC & S & S & S & S & S & S & S & This study \\
\hline 29 & Heinz 1706 & Inbred line & TGRC & S & S & S & S & S & S & S & This study \\
\hline 30 & New Yorker & Inbred line & TGRC & S & S & S & S & S & $\mathrm{R}$ & S & Jung et al. (2014) \\
\hline 31 & Miniheuksu & $\mathrm{F}_{1}$ hybrid & Asia Seed Co., Ltd. & $\mathrm{R}$ & $\mathrm{S}$ & S & $\mathrm{S}$ & $\mathrm{S}$ & $\mathrm{R}$ & $\mathrm{S}$ & Kim et al. (2018) \\
\hline 32 & TY Unique & $\mathrm{F}_{1}$ hybrid & Asia Seed Co., Ltd. & $\mathrm{R}$ & S & S & S & $\mathrm{H}$ & $\mathrm{R}$ & $\mathrm{H}$ & Supplier \\
\hline 33 & Shinsugar Yellow & $\mathrm{F}_{1}$ hybrid & Asia Seed Co., Ltd. & $\mathrm{H}$ & $\mathrm{H}$ & $\mathrm{H}$ & $\mathrm{H}$ & $\mathrm{H}$ & $\mathrm{R}$ & $\mathrm{H}$ & Supplier \\
\hline 34 & Red Strong & $\mathrm{F}_{1}$ hybrid & Bunong Seed & $\mathrm{R}$ & S & $\mathrm{R}$ & $\mathrm{R}$ & S & $\mathrm{R}$ & $\mathrm{R}$ & This study \\
\hline 35 & Sun Star & $\mathrm{F}_{1}$ hybrid & Bunong Seed & $\mathrm{H}$ & S & S & S & S & $\mathrm{H}$ & S & Supplier \\
\hline 36 & Black Eagle & $\mathrm{F}_{1}$ hybrid & Bunong Seed & $\mathrm{R}$ & S & $\mathrm{H}$ & $\mathrm{H}$ & S & S & $\mathrm{H}$ & Supplier \\
\hline 37 & Tamla & $\mathrm{F}_{1}$ hybrid & Bunong Seed & $\mathrm{H}$ & S & $\mathrm{H}$ & $\mathrm{H}$ & S & $\mathrm{H}$ & S & Supplier \\
\hline 38 & Bntoskna & $\mathrm{F}_{1}$ hybrid & Bunong Seed & $\mathrm{H}$ & S & S & $\mathrm{H}$ & $\mathrm{S}$ & $\mathrm{H}$ & $\mathrm{H}$ & Supplier \\
\hline 39 & TY Izzang & $\mathrm{F}_{1}$ hybrid & Bunong Seed & $\mathrm{H}$ & S & $\mathrm{H}$ & $\mathrm{H}$ & S & $\mathrm{R}$ & S & Supplier \\
\hline 40 & Candy Plus & $\mathrm{F}_{1}$ hybrid & Bunong Seed & $\mathrm{H}$ & S & $\mathrm{H}$ & $\mathrm{H}$ & S & $\mathrm{H}$ & S & Supplier \\
\hline 41 & Super Star & $\mathrm{F}_{1}$ hybrid & Bunong Seed & $\mathrm{H}$ & S & S & S & S & $\mathrm{H}$ & $\mathrm{H}$ & Supplier \\
\hline 42 & Red Zenith & $\mathrm{F}_{1}$ hybrid & Bunong Seed & $\mathrm{H}$ & S & $\mathrm{H}$ & $\mathrm{H}$ & $\mathrm{S}$ & $\mathrm{R}$ & $\mathrm{H}$ & Supplier \\
\hline 43 & TY Hunter & $\mathrm{F}_{1}$ hybrid & Bunong Seed & $\mathrm{R}$ & S & $\mathrm{H}$ & $\mathrm{H}$ & S & $\mathrm{H}$ & $\mathrm{H}$ & Supplier \\
\hline 44 & TY One Top & $\mathrm{F}_{1}$ hybrid & Bunong Seed & S & S & S & $\mathrm{H}$ & S & $\mathrm{H}$ & $\mathrm{H}$ & Supplier \\
\hline 45 & Yureka & $\mathrm{F}_{1}$ hybrid & Bunong Seed & S & S & $\mathrm{H}$ & $\mathrm{H}$ & S & $\mathrm{H}$ & $\mathrm{H}$ & Supplier \\
\hline 46 & Black Ace & $\mathrm{F}_{1}$ hybrid & Bunong Seed & $\mathrm{R}$ & S & S & S & S & S & $\mathrm{H}$ & Supplier \\
\hline 47 & Oasis & $\mathrm{F}_{1}$ hybrid & Bunong Seed & S & S & $\mathrm{H}$ & S & S & $\mathrm{H}$ & S & Supplier \\
\hline 48 & TY Megaton & $\mathrm{F}_{1}$ hybrid & Bunong Seed & S & S & S & S & S & $\mathrm{R}$ & S & Supplier \\
\hline 49 & Gold Sugar & $\mathrm{F}_{1}$ hybrid & Bunong Seed & $\mathrm{R}$ & S & $\mathrm{H}$ & $\mathrm{H}$ & $\mathrm{R}$ & $\mathrm{R}$ & $\mathrm{S}$ & Supplier \\
\hline 50 & Dotaerang Red & $\mathrm{F}_{1}$ hybrid & Dong seo seed & $\mathrm{H}$ & $\mathrm{H}$ & S & S & S & $\mathrm{R}$ & S & This study \\
\hline 51 & Black Ball & $\mathrm{F}_{1}$ hybrid & Dongoh Seed & $\mathrm{R}$ & S & $\mathrm{H}$ & $\mathrm{H}$ & S & $\mathrm{H}$ & $\mathrm{H}$ & Supplier \\
\hline 52 & Kolmi & $\mathrm{F}_{1}$ hybrid & Dongoh Seed & S & S & $\mathrm{H}$ & $\mathrm{R}$ & $\mathrm{H}$ & $\mathrm{R}$ & S & Supplier \\
\hline 53 & Sky Ball & $\mathrm{F}_{1}$ hybrid & Dongoh Seed & $\mathrm{R}$ & S & $\mathrm{H}$ & $\mathrm{H}$ & S & $\mathrm{R}$ & $\mathrm{R}$ & Supplier \\
\hline 54 & Starbuck & $\mathrm{F}_{1}$ hybrid & Farm Hannong & $\mathrm{R}$ & S & S & S & S & S & S & Supplier \\
\hline 55 & Olleh TY & $\mathrm{F}_{1}$ hybrid & Farm Hannong & S & S & $\mathrm{H}$ & $\mathrm{H}$ & S & $\mathrm{R}$ & S & Supplier \\
\hline 56 & Rafito & $\mathrm{F}_{1}$ hybrid & Farm Hannong & $\mathrm{H}$ & S & $\mathrm{S}$ & S & $\mathrm{S}$ & $\mathrm{H}$ & $\mathrm{R}$ & Supplier \\
\hline 57 & Big Wonderful & $\mathrm{F}_{1}$ hybrid & Gonong Seed & $\mathrm{R}$ & S & $\mathrm{H}$ & $\mathrm{H}$ & S & $\mathrm{H}$ & S & Supplier \\
\hline 58 & TY Carnival & $\mathrm{F}_{1}$ hybrid & Gyeongwon & $\mathrm{R}$ & S & $\mathrm{H}$ & $\mathrm{H}$ & S & $\mathrm{H}$ & $\mathrm{H}$ & Supplier \\
\hline 59 & Legend Summer & $\mathrm{F}_{1}$ hybrid & Haesung Seed Plus & S & $\mathrm{S}$ & S & $\mathrm{S}$ & $\mathrm{S}$ & $\mathrm{R}$ & S & Supplier \\
\hline 60 & Daewang & $\mathrm{F}_{1}$ hybrid & Jeil Seed Bio & $\mathrm{H}$ & S & S & S & S & S & S & Supplier \\
\hline
\end{tabular}


Table 4. (continued)

\begin{tabular}{|c|c|c|c|c|c|c|c|c|c|c|c|}
\hline \multirow{2}{*}{ No. } & \multirow{2}{*}{ Tomato cultivar/line } & \multirow{2}{*}{ Type } & \multirow{2}{*}{ Company/Supplier ${ }^{a}$} & \multicolumn{7}{|c|}{ SNP marker genotype ${ }^{b}$} & \multirow{2}{*}{ Reference $^{c}$} \\
\hline & & & & RsR6-1 & RsR6-2 & RsR6-3 & RsR6-4 & RsR6-5 & RsR6-6 & RsR12-1 & \\
\hline 61 & Hongboseok & $\mathrm{F}_{1}$ hybrid & Jeil Seed Bio & S & S & S & S & S & S & S & Supplier \\
\hline 62 & Jeilheukjinju & $F_{1}$ hybrid & Jeil Seed Bio & $\mathrm{S}$ & $\mathrm{S}$ & $\mathrm{S}$ & $\mathrm{R}$ & $\mathrm{S}$ & $\mathrm{R}$ & $\mathrm{S}$ & Supplier \\
\hline 63 & Dotaerang Myeongpum & $\mathrm{F}_{1}$ hybrid & Jeil Seed Bio & S & S & S & S & S & S & S & Supplier \\
\hline 64 & Heukryong & $F_{1}$ hybrid & Jeil Seed Bio & S & S & S & S & S & S & $\mathrm{H}$ & Supplier \\
\hline 65 & Minijaok & $F_{1}$ hybrid & Jeil Seed Bio & S & S & S & $\mathrm{R}$ & S & $\mathrm{R}$ & S & Supplier \\
\hline 66 & Sinheukjinju & $F_{1}$ hybrid & Jeil Seed Bio & $\mathrm{H}$ & $\mathrm{R}$ & S & $\mathrm{R}$ & $\mathrm{R}$ & $\mathrm{R}$ & S & Supplier \\
\hline 67 & Super Dotaerang & $\mathrm{F}_{1}$ hybrid & Koregon seed & $\mathrm{H}$ & $\mathrm{H}$ & S & S & S & $\mathrm{R}$ & S & This study \\
\hline 68 & Lezaforta $\mathrm{F}_{1}$ & $\mathrm{~F}_{1}$ hybrid & Mifko seed & S & S & S & S & S & $\mathrm{H}$ & S & Supplier \\
\hline 69 & Unicorn & $\mathrm{F}_{1}$ hybrid & Monsanto Korea & $\mathrm{H}$ & S & S & S & S & $\mathrm{R}$ & S & Supplier \\
\hline 70 & SV $7160 \mathrm{TC}$ & $\mathrm{F}_{1}$ hybrid & Monsanto Korea & $\mathrm{R}$ & S & $\mathrm{H}$ & $\mathrm{R}$ & $\mathrm{R}$ & $\mathrm{R}$ & S & Supplier \\
\hline 71 & SV02444 TG & $F_{1}$ hybrid & Monsanto Korea & S & S & $\mathrm{H}$ & S & S & $\mathrm{R}$ & $\mathrm{H}$ & Supplier \\
\hline 72 & Bacchus & $F_{1}$ hybrid & Monsanto Korea & $\mathrm{R}$ & $\mathrm{S}$ & $\mathrm{H}$ & $\mathrm{H}$ & S & $\mathrm{R}$ & $\mathrm{S}$ & Supplier \\
\hline 73 & SV4224 TH & $F_{1}$ hybrid & Monsanto Korea & S & S & $\mathrm{H}$ & S & S & $\mathrm{R}$ & $\mathrm{H}$ & Supplier \\
\hline 74 & SV0339TG & $\mathrm{F}_{1}$ hybrid & Monsanto Korea & S & S & $\mathrm{H}$ & S & S & $\mathrm{R}$ & $\mathrm{H}$ & Supplier \\
\hline 75 & Tiara & $F_{1}$ hybrid & Nongwoo Bio Co., Ltd. & $\mathrm{H}$ & S & S & S & S & $\mathrm{R}$ & S & Kim et al. (2018) \\
\hline 76 & TY SenseQ & $F_{1}$ hybrid & Nongwoo Bio Co., Ltd. & S & S & $\mathrm{H}$ & $\mathrm{H}$ & S & $\mathrm{H}$ & S & Supplier \\
\hline 77 & Redpang & $\mathrm{F}_{1}$ hybrid & Nongwoo Bio Co., Ltd. & S & S & S & S & S & $\mathrm{R}$ & S & Supplier \\
\hline 78 & Titichal & $\mathrm{F}_{1}$ hybrid & Nongwoo Bio Co., Ltd. & S & S & $\mathrm{H}$ & $\mathrm{H}$ & S & $\mathrm{R}$ & S & Supplier \\
\hline 79 & TY Altorang & $F_{1}$ hybrid & Nongwoo Bio Co., Ltd. & S & S & $\mathrm{H}$ & $\mathrm{H}$ & S & $\mathrm{R}$ & S & Supplier \\
\hline 80 & Beta Tiny & $F_{1}$ hybrid & Nongwoo Bio Co., Ltd. & S & S & S & $\mathrm{H}$ & $\mathrm{H}$ & $\mathrm{H}$ & S & Supplier \\
\hline 81 & TY Tiny & $\mathrm{F}_{1}$ hybrid & Nongwoo Bio Co., Ltd. & S & S & $\mathrm{H}$ & $\mathrm{H}$ & S & $\mathrm{H}$ & S & Supplier \\
\hline 82 & Cupirang & $F_{1}$ hybrid & Nongwoo Bio Co., Ltd. & S & S & S & S & S & $\mathrm{R}$ & S & Supplier \\
\hline 83 & Minichal & $\mathrm{F}_{1}$ hybrid & Nongwoo Bio Co., Ltd. & S & S & S & $\mathrm{H}$ & $\mathrm{H}$ & $\mathrm{H}$ & S & Supplier \\
\hline 84 & TY Sispen & $F_{1}$ hybrid & Nongwoo Bio Co., Ltd. & $\mathrm{S}$ & $\mathrm{S}$ & $\mathrm{H}$ & $\mathrm{H}$ & $\mathrm{S}$ & $\mathrm{R}$ & $\mathrm{S}$ & Supplier \\
\hline 85 & Black Change & $F_{1}$ hybrid & Nongwoo Bio Co., Ltd. & $\mathrm{H}$ & S & $\mathrm{H}$ & $\mathrm{H}$ & S & $\mathrm{H}$ & $\mathrm{H}$ & Supplier \\
\hline 86 & Mulya & $F_{1}$ hybrid & RDA & S & $\mathrm{S}$ & S & S & S & $\mathrm{R}$ & S & Kim et al. (2018) \\
\hline 87 & Sigyo 1 ho & $F_{1}$ hybrid & RDA & S & S & S & S & S & $\mathrm{R}$ & S & Kim et al. (2018) \\
\hline 88 & Broadley & $F_{1}$ hybrid & RDA & $\mathrm{R}$ & S & S & S & S & $\mathrm{R}$ & S & Kim et al. (2018) \\
\hline 89 & Yulwon & $F_{1}$ hybrid & RDA & S & $\mathrm{H}$ & $\mathrm{H}$ & $\mathrm{R}$ & $\mathrm{H}$ & $\mathrm{R}$ & S & Kim et al. (2018) \\
\hline 90 & Hoyong & $F_{1}$ hybrid & Sakata Korea & $\mathrm{H}$ & S & S & S & S & $\mathrm{R}$ & $\mathrm{H}$ & Kim et al. (2018) \\
\hline 91 & Tosama & $\mathrm{F}_{1}$ hybrid & Sakata Korea & S & S & S & $\mathrm{H}$ & S & $\mathrm{H}$ & $\mathrm{S}$ & Supplier \\
\hline 92 & Super Sun Road & $\mathrm{F}_{1}$ hybrid & Sakata Korea & $\mathrm{H}$ & S & S & $\mathrm{H}$ & S & $\mathrm{H}$ & $\mathrm{H}$ & Supplier \\
\hline 93 & Super Top & $\mathrm{F}_{1}$ hybrid & Sakata Korea & S & S & S & S & S & $\mathrm{H}$ & $\mathrm{H}$ & Supplier \\
\hline 94 & Lokousan Maru & $\mathrm{F}_{1}$ hybrid & Sakata Korea & S & S & S & S & S & $\mathrm{H}$ & S & Supplier \\
\hline 95 & Taiyau & $F_{1}$ hybrid & Sakata Korea & S & S & S & S & S & $\mathrm{H}$ & $\mathrm{H}$ & Supplier \\
\hline 96 & Taihu & $F_{1}$ hybrid & Sakata Korea & $\mathrm{R}$ & S & $\mathrm{H}$ & S & S & $\mathrm{H}$ & $\mathrm{H}$ & Supplier \\
\hline 97 & Super Top & $F_{1}$ hybrid & Sakata Korea & S & S & S & S & S & $\mathrm{H}$ & $\mathrm{H}$ & Supplier \\
\hline 98 & Tiger & $F_{1}$ hybrid & Samsung Seeds & $\mathrm{H}$ & S & S & S & S & $\mathrm{R}$ & S & Supplier \\
\hline 99 & Chalstone TY & $F_{1}$ hybrid & Sky seed & S & S & $\mathrm{H}$ & $\mathrm{H}$ & S & $\mathrm{R}$ & $\mathrm{H}$ & Supplier \\
\hline 100 & TY Marathon & $\mathrm{F}_{1}$ hybrid & Sky seed & S & S & S & S & S & $\mathrm{R}$ & $\mathrm{H}$ & Supplier \\
\hline 101 & Rapsody & $F_{1}$ hybrid & Syngenta Korea & $\mathrm{H}$ & S & S & $\mathrm{H}$ & S & S & S & Supplier \\
\hline 102 & Madison & $F_{1}$ hybrid & Syngenta Korea & $\mathrm{H}$ & S & S & S & S & S & S & Supplier \\
\hline 103 & Ricophin-9 & $F_{1}$ hybrid & Syngenta Korea & $\mathrm{H}$ & S & S & $\mathrm{H}$ & $\mathrm{H}$ & $\mathrm{R}$ & S & Supplier \\
\hline 104 & Duine & $\mathrm{F}_{1}$ hybrid & Syngenta Korea & $\mathrm{H}$ & S & S & S & S & $\mathrm{H}$ & S & Supplier \\
\hline 105 & Dafnis & $F_{1}$ hybrid & Syngenta Korea & $\mathrm{S}$ & $\mathrm{S}$ & $\mathrm{H}$ & $\mathrm{H}$ & S & $\mathrm{H}$ & $\mathrm{H}$ & Supplier \\
\hline 106 & Komodo & $\mathrm{F}_{1}$ hybrid & Syngenta Korea & S & S & $\mathrm{H}$ & $\mathrm{H}$ & S & $\mathrm{H}$ & $\mathrm{H}$ & Supplier \\
\hline 107 & Tory & $\mathrm{F}_{1}$ hybrid & Syngenta Korea & S & S & $\mathrm{H}$ & $\mathrm{H}$ & S & $\mathrm{H}$ & $\mathrm{H}$ & Supplier \\
\hline 108 & Mamirio & $F_{1}$ hybrid & Syngenta Korea & $\mathrm{R}$ & $\mathrm{S}$ & $\mathrm{H}$ & $\mathrm{H}$ & S & $\mathrm{H}$ & $\mathrm{H}$ & Supplier \\
\hline 109 & European Rapsodie & $F_{1}$ hybrid & Syngenta Korea & $\mathrm{H}$ & S & $\mathrm{S}$ & $\mathrm{H}$ & S & S & S & Supplier \\
\hline 110 & Trio Plus & $\mathrm{F}_{1}$ hybrid & Taeyang seed & $\mathrm{R}$ & S & $\mathrm{H}$ & $\mathrm{H}$ & S & $\mathrm{R}$ & $\mathrm{H}$ & Supplier \\
\hline 111 & Kang Jeok & $\mathrm{F}_{1}$ hybrid & Taeyang seed & $\mathrm{R}$ & S & $\mathrm{H}$ & $\mathrm{H}$ & S & $\mathrm{H}$ & S & Supplier \\
\hline 112 & Dotaerang TY Winner & $\mathrm{F}_{1}$ hybrid & Takii Korea & S & S & $\mathrm{H}$ & S & S & $\mathrm{R}$ & S & Supplier \\
\hline 113 & Doterang Plus & $\mathrm{F}_{1}$ hybrid & Takii Korea & S & S & S & S & S & $\mathrm{R}$ & S & Supplier \\
\hline 114 & Dotaerang Solar & $\mathrm{F}_{1}$ hybrid & Takii Korea & S & S & S & S & S & $\mathrm{R}$ & $\mathrm{H}$ & Supplier \\
\hline 115 & Cuty & $\mathrm{F}_{1}$ hybrid & Takii Korea & S & $\mathrm{H}$ & $\mathrm{H}$ & $\mathrm{H}$ & $\mathrm{H}$ & $\mathrm{R}$ & S & Supplier \\
\hline 116 & Dotaerang Diamond & $F_{1}$ hybrid & Takii Korea & S & S & S & S & S & $\mathrm{R}$ & S & Supplier \\
\hline 117 & LA1589 & Wild species & TGRC & $\mathrm{R}$ & S & $\mathrm{R}$ & $\mathrm{R}$ & $\mathrm{R}$ & $\mathrm{R}$ & S & This study \\
\hline
\end{tabular}

${ }^{a}$ Company/supplier of seed or DNA sample: KHU = Kyung Hee University, RDA = National Agrobiodiversity Center (RDA-Genebank), TGRC = Tomato Genetics Resource Center. All tomato genotypes belong to Solanum lycopersicum species except LA1589 (Solanum pimpinellifolium).

${ }^{b} \mathrm{SNP}$ marker genotype: $\mathrm{R}=$ resistant, $\mathrm{S}=$ susceptible, $\mathrm{H}=$ heterozygous.

${ }^{c}$ Reference for the phenotypic information of the tomato cultivars used in the study. Supplier's phenotypic information was obtained via personal communication from the company. 
B

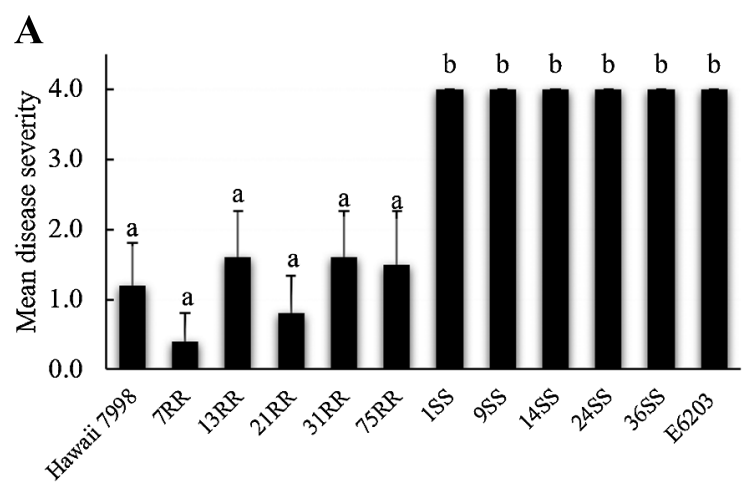

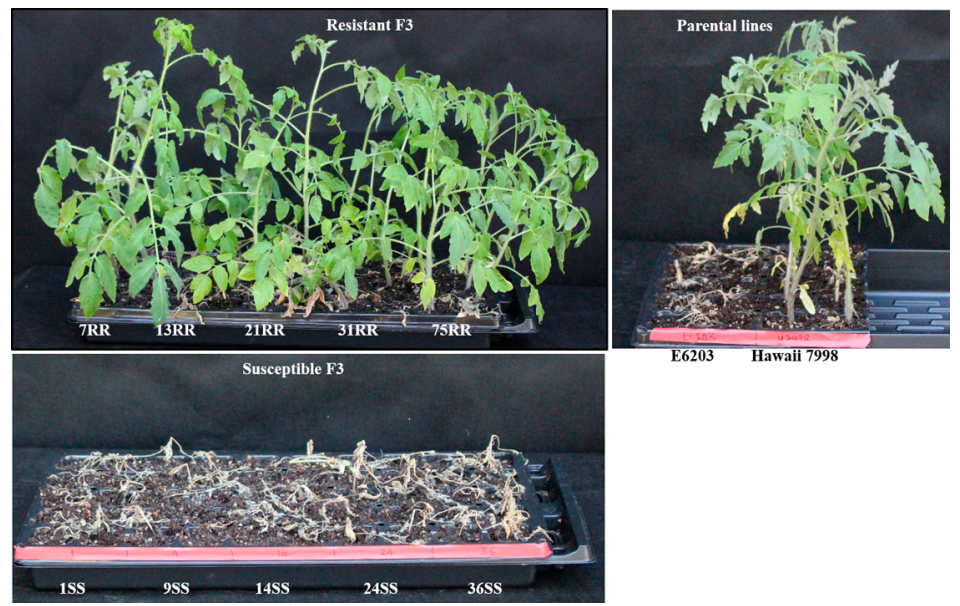

Fig. 3. Evaluation of $\mathrm{F}_{3}$ generation selected by RsR6-5 and RsR12-1 for resistance to R. pseudosolanacearum. (A) Mean disease severity of $\mathrm{F}_{3}$ generation developed from a crossing between E6203 and Hawaii 7998 one month after inoculation with $R$. pseudosolanacearum strain SL882. The genotypes of the $\mathrm{F}_{3}$ lines are either homozygous resistant or susceptible at RsR6-5 and RsR12-1. The $\mathrm{F}_{3}$ lines are followed by RR (carrying resistant alleles of both Bwr6 and Bwr-12) or SS (carrying susceptible alleles at both loci). Different letters on the bars indicate a significant difference $(P<0.05)$ of mean disease severity. (B) Photographs of homozygous $\mathrm{F}_{3}$ generation and parental lines one month after inoculation with SL882.

or heterozygous genotype to RsR12-1 exhibited a resistant phenotype. However, homozygous susceptible or heterozygous genotypes with RsR6-5 exhibited a susceptible phenotype regardless of the RsR12-1 genotype. One exception to this was High Power, which exhibits a resistant phenotype although it has a heterozygous genotype with RsR6-5. The genotype of RsR6-5 is highly correlated with the bacterial wilt phenotype of tomato cultivars and selected for tracing $B w r-6$. The diagnostic accuracy of the markers was evaluated and RsR6-5 and RsR12-1 combination was resulted in $94.1 \%$ true positive rate and $100 \%$ true negative rate (Supplemental Table 1). Taken together, RsR6-5 and RsR12-1 should be used for effective marker-assisted selection of bacterial wilt resistance in tomatoes

\section{Validation of the RsR6-5 and RsR12-1 markers using $F_{3}$ populations}

To validate the efficiency of the two markers for selecting resistant lines in the segregating population, $10 \mathrm{~F}_{3}$ generations homozygous resistant and susceptible (each five lines), and eight $F_{3}$ generation carrying only Bwr-6 or Bwr-12 (each four lines) were developed from E6203 (susceptible) and Hawaii 7998 (resistant) were selected based on RsR6-5 and RsR12-1 genotype. Hawaii 7998 is one of the entries in international set of bacterial wilt resistant lines evaluated in twelve fields and showed an average of $90 \%$ survival rate (Wang et al. 1998). The resistance of Hawaii 7998 and Hawaii 7996 was derived from the same origin, PI 127805A (S. pimpinellifolium) (Daunay et al. 2010, Scott et al. 2005). Hence, genetic resistance to bacterial wilt in these two lines might be governed by same gene.

Ten plants of each $F_{3}$ generation, along with the two parental lines, were inoculated with $R$. pseudosolanacearum strain SL882 for disease evaluation. The two parental lines exhibited distinct differences in bacterial wilt resistance as expected, with mean disease scores of $4.0 \pm 0.0$ and $1.2 \pm 0.61$, respectively. The mean disease severity between homozygous resistant and susceptible genotypes in the $F_{3}$ generation was significantly different (Fig. 3A). Lines with homozygous resistant genotypes exhibited highly resistant phenotypes with mean disease severity scores ranging from $0.4 \pm 0.40$ to $1.6 \pm 0.65$, which were not significantly different from that of the resistant parent. On the other hand, homozygous susceptible $\mathrm{F}_{3}$ exhibited highly susceptible phenotypes with mean disease severity scores of $4.0 \pm 0.0$, which were similar to the susceptible parent (Fig. 3A, 3B). $\mathrm{F}_{3}$ progenies carrying resistant allele only in RsR6-5 or RsR12-1 were susceptible (Supplemental Fig. 1). These results suggest that the combination of RsR6-5 and RsR12-1, which are associated with Bwr-6 and Bwr-12, respectively, are predictive for bacterial wilt resistance.

\section{Discussion}

Genetic resistance is the most effective control strategy for bacterial wilt of tomato, and multiple breeding programs have been engaged in developing resistant lines by incorporating resistance from different resistant sources (Daunay et al. 2010, Wang et al. 1998). Genomic regions linked to bacterial wilt resistance in the well-known resistant cultivar, Hawaii 7996, were identified on different chromosomes, and some regions were detected against specific strains (Thoquet et al. 1996a, 1996b, Wang et al. 2000). $B w r-3$ and Bwr-4 were associated with resistance against phylotype II strains, while $B w r-12$ was specific to 
Phylotype-I (Carmeille et al. 2006). In contrast, Bwr-6 was associated with various strains from different phylotypes (I and II) (Thoquet et al. 1996a) and consistently detected under various conditions (Carmeille et al. 2006, Geethanjali et al. 2010, Mangin et al. 1999, Wang et al. 2013). Genetic analysis of bacterial wilt resistance in S. lycopersicum var. cerasiforme 'L285' identified three QTL on chromosomes 6, 7, and 10 (Danesh et al. 1994). The genomic regions associated with bacterial wilt resistance on chromosome 6 in both Hawaii 7996 and L285 were colocalized. Furthermore, the most stable QTL for bacterial wilt resistance in eggplant was also identified in chromosome 6 and syntenic with Bwr-6 of tomato (Salgon et al. 2018). All these suggest the significance of Bwr-6 in bacterial wilt resistance in tomato and likely in other Solanaceae crops. The development of functional markers for such broad-spectrum QTL is essential to facilitate markerassisted breeding.

Genetic analysis using only segregating populations is time-consuming, cost-inefficient, and the developed markers may be specific to certain resistant lines (Pascual et al. 2016). In this regard, we used a complementary approach to dissect the Bwr-6 region, using germplasm collections and $\mathrm{F}_{3}$ generation to validate our result. SNP-based CAPS/ dCAPS markers near Bwr-6 were developed and validated. A total of 117 tomato germplasms were screened with newly developed markers for Bwr-6 genotypes, and the corresponding phenotypic information was used to explore the efficiency of each marker.

Among 17 resistant cultivars used to screen the markers in the Bwr-6 region, four cultivars (IT 201664, High Power, Super High Power, and Spider) had susceptible genotypes with RsR6-1, RsR6-2, and RsR6-3. On the other hand, all resistant cultivars had homozygous resistant genotypes with RsR6-4, RsR6-5, and RsR6-6 except High Power, which has a heterozygous genotype with RsR6-4 and RsR6-5. Similarly, all cultivars exhibiting resistant phenotypes have homozygous resistant genotypes with RsR12-1, except BBlocking, Shincheonggang, Fighting, and Geumgang which are heterozygous to this marker. The true positive rate of RsR6-1, RsR6-2, and RsR6-3 combined with RsR12-1 was $47.1 \%$ whereas that of RsR6-4 and RsR6-5 was 94.1\% and that of RsR6-6 was $100 \%$. Based on these observations, we hypothesized that the three markers (RsR6-4, RsR6-5, and RsR6-6) are better predictors of resistance conferred by Bwr-6 than RsR6-1, RsR6-2, and RsR6-3.

The markers were further compared using the susceptible panel of germplasms. Some cultivars exhibiting susceptible phenotypes, such as Red Strong, are resistant to RsR6-4, RsR6-6, and RsR12-1. However, all cultivars having homozygous resistant genotypes with RsR6-5 and RsR12-1 exhibited resistant phenotypes. In summary, $99 \%$ and $87 \%$ true negative rate was obtained for RsR6-4 and RsR6-6 while RsR6-5 resulted in $100 \%$ true negative rate genotypes in combination with RsR12-1 (Supplemental Table 1). These results suggest that RsR6-5 is the best diag- nostic marker to trace $B w r-6$ associated with bacterial wilt resistance. Diagnostic markers developed based only on a segregating population may not fully correlate with the trait when tested in diverse germplasms, hindering their utilization for marker-assisted selection in a broad set of breeding germplasms (Niewohner et al. 1995). Therefore, utilization of a wide range of germplasms, including inbred lines, commercial $F_{1}$ hybrids, and wild species, for validating developed markers is essential before deployment to endusers, including breeders and farmers.

The diagnostic potential of RsR6-5 coupled with RsR12-1 for bacterial wilt resistance in tomato was tested using a broader set of germplasms (Bartkiewicz et al. 2018, Yang et al. 2015) and can be used for marker-assisted selection in commercial breeding programs. Without determining the genotyping results for another major resistance QTL, Bwr-12, determination of Bwr-6 genotype with the RsR6-5 marker alone is able to predict resistant and susceptible phenotypes in the tomato cultivars used in this study with $94.1 \%$ and $96 \%$ accuracy, respectively. High Power, SV7160TC, Gold Sugar, Sinheukjinju, and LA1589 showed non-matching genotypes. On the other hand, Bwr-12 genotyping determined by RsR12-1 alone was able to predict resistant and susceptible phenotypes with $100 \%$ and $66 \%$ accuracy, respectively. Resistance conferred by the Bwr-12 genotype shows a dominant inheritance pattern (Kim et al. 2018). RsR6-5 and RsR12-1 combination was resulted in $94.1 \%$ of true positive rate and $100 \%$ true negative rate showing the highest diagnostic accuracy compared to other marker combinations.

Heterozygous to RsR6-5 and either heterozygous or homozygous resistant to RsR12-1 yielded susceptible phenotypes except for High Power, which suggests that the Bwr-6 resistance allele might be recessive; however, this should be further validated using a segregating population. Recessive gene resistance to bacterial disease has been reported in Arabidopsis and rice. $R$. solanacearum resistance in Arabidopsis thaliana is governed by a recessively inherited gene (RRS1-R) (Deslandes et al. 2002). Similarly, among more than 43 resistance genes identified so far for bacterial blight in rice caused by Xanthomonas oryzae pv. oryzae, 16 are inherited as recessive traits (Kim 2018, Vikal and Bhatia 2017).

Extensive QTL mapping studies have been conducted to identify genomic regions associated with bacterial wilt resistance (Carmeille et al. 2006, Geethanjali et al. 2010, Mangin et al. 1999, Thoquet et al. 1996a, 1996b, Wang et al. 2000, 2013). The release of the tomato reference genome (Tomato Genome Consortium 2012) and availability of whole-genome resequencing data for various tomato cultivars (Lin et al. 2014, The 100 Tomato Genome Sequencing Consortium 2014) facilitated comparisons among tomato genotypes using genome-wide SNP markers for different traits. The adequate number of SNPs among tomato genotypes can be used to saturate markers nearby previously identified QTL regions. Accordingly, whole-genome 
resequencing of bacterial wilt resistant and susceptible tomato cultivars revealed genome-wide SNPs that were candidates for distinguishing the two groups of tomato, with the highest number of non-synonymous SNPs identified on chromosomes 12 and 6 (Kim et al. 2018). Analysis of SNPs near Bwr-12 in the same study discovered molecular marker (KHU-1) tightly linked to bacterial wilt resistance; this marker was used to discriminate resistant and susceptible tomato cultivars. The analysis of SNPs near $B w r-6$ and the development of diagnostic markers in this study will pave the way toward identifying candidate genes and facilitating resistance gene pyramiding.

RsR6-5 is located in the coding region of Solyc06g054230.2, which encodes a putative calmodulin protein kinase, suggesting that this gene could be a possible candidate gene for bacterial wilt resistance in tomato. The nucleotide substitution of guanine to thymine at $128 \mathrm{bp}$ changes the amino acid aspartate to glutamate. The majority of plant disease resistance genes encode nucleotidebinding site-leucine-rich repeat (NBS-LRR) proteins (McHale et al. 2006). However, the role of calmodulin proteins in the response of plants to biotic and abiotic stresses has also been reported (Cheval et al. 2013, Zeng et al. 2015). Calmodulin is a calcium-binding protein and regulates downstream calcium signal-related responses. The expression of tomato calmodulin genes is significantly altered upon pathogen infection. Functional analysis revealed that the silencing of SlCaM2 (Solyc10g081170.1.1) and SlCaM6 (Solyc03g098050.2.1) in tomato reduced its resistance to tomato rattle virus and Pythium aphanidermatum and decreased the expression of downstream signaling and defense-related genes (Zhao et al. 2013). Transcriptome analysis of bacterial wilt-resistant (LS-89) and susceptible (Ponderosa) cultivars indicated an approximately 30 -fold increase of a putative calmodulin-binding family protein in response to $R$. solanacearum infection in resistant cultivars, while the analogous response in susceptible cultivars was very limited (Ishihara et al. 2012). These findings suggest that Solyc06g054230.2 may play an important role in bacterial wilt resistance in tomato; although, further analysis is required to elucidate the gene function.

In conclusion, SNPs near Bwr-6 were analyzed to search for markers tightly linked to this QTL. A total of 117 tomato germplasms were used to validate newly developed markers near this QTL. Among the analyzed markers, RsR6-5 is tightly linked to bacterial wilt resistance derived from Bwr-6. Consequently, this marker, in combination with RsR12-1, effectively predicted bacterial wilt-resistant and susceptible cultivars. The significance of these markers was further validated using an $\mathrm{F}_{3}$ generation developed from a crossing between resistant and susceptible parents. $\mathrm{F}_{3}$ lines that had resistant genotypes with RsR6-5 and RsR12-1 exhibited resistant phenotypes, while susceptible to the same markers exhibited susceptible phenotypes. The SNP-based diagnostic marker to Bwr-6 was not identified in previous studies, and the newly developed marker in this study (RsR6-5) will help to trace this locus in markerassisted breeding of tomato cultivars that are resistant to the devastating effects of bacterial wilt.

\section{Author Contribution Statement}

JML conceived and designed the experiments. AMA, JC and YK performed the experiments. CSO, IY, and ISN provided experimental materials. AMA and JML wrote and revised the manuscript, and all co-authors contributed and approved the final draft of the manuscript.

\section{Acknowledgment}

This work was supported by the Golden Seed Project (Center for Horticultural Seed Development) funded by the Ministry of Agriculture, Food and Rural Affairs of Korea.

\section{Literature Cited}

Abebe, A.M., K.P.P. Wai, M.I. Siddique, H.-S. Mo, H.J. Yoo, Y. Jegal, S.-E. Byeon, K.-S. Jang, S.-G. Jeon, J.-E. Hwang et al. (2016) Evaluation of Phytophthora root rot- and bacterial wilt-resistant inbred lines and their crosses for use as rootstocks in pepper (Capsicum annuum L.). Hortic. Environ. Biotechnol. 57: 598-605.

Bae, C., S.W. Han, Y.R. Song, B.Y. Kim, H.J. Lee, J.M. Lee, I. Yeam, S. Heu and C.S. Oh (2015) Infection processes of xylemcolonizing pathogenic bacteria: possible explanations for the scarcity of qualitative disease resistance genes against them in crops. Theor. Appl. Genet. 128: 1219-1229.

Bartkiewicz, A., F. Chilla, D. Terefe-Ayana, J. Lübeck, J. Strahwald, E. Tacke, H.-R. Hofferbert, K. Flath, M. Linde and T. Debener (2018) Improved genetic resolution for linkage mapping of resistance to potato wart in monoparental dihaploids with potential diagnostic value in tetraploid potato varieties. Theor. Appl. Genet. 131: 2555-2566.

Caldwell, D., B.S. Kim and A.S. Iyer-Pascuzzi (2017) Ralstonia solanacearum differentially colonizes roots of resistant and susceptible tomato plants. Phytopathology 107: 528-536.

Carmeille, A., C. Caranta, J. Dintinger, P. Prior, J. Luisetti and P. Besse (2006) Identification of QTLs for Ralstonia solanacearum race 3-phylotype II resistance in tomato. Theor. Appl. Genet. 113: $110-121$.

Cheval, C., D. Aldon, J.-P. Galaud and B. Ranty (2013) Calcium/ calmodulin-mediated regulation of plant immunity. Biochim. Biophys. Acta 1833: 1766-1771.

Cho, H., E.S. Song, Y.K. Lee, S. Lee, S.W. Lee, A. Jo, B.M. Lee, J.G. Kim and I. Hwang (2018) Analysis of genetic and pathogenic diversity of Ralstonia solanacearum causing potato bacterial wilt in Korea. Plant Pathol. J. 34: 23-34.

Danesh, D., S. Aarons, G.E. McGill and N.D. Young (1994) Genetic dissection of oligogenic resistance to bacterial wilt in tomato. Mol. Plant Microbe Interact. 7: 464-471.

Daunay, M.C., H. Laterrot, J.W. Scott, P. Hanson and J.-F. Wang (2010) Tomato resistance to bacterial wilt caused by Ralstonia solanaearum E.F. Smith: ancestry and peculiarities. In: Scott, J.W. (ed.) Report of the Tomato Genetics Cooperative, University of Florida, Wimauma, FL 33598 USA.

Deslandes, L., J. Olivier, F. Theulieres, J. Hirsch, D.X. Feng, P. 
Bittner-Eddy, J. Beynon and Y. Marco (2002) Resistance to Ralstonia solanacearum in Arabidopsis thaliana is conferred by the recessive $R R S 1-R$ gene, a member of a novel family of resistance genes. Proc. Natl. Acad. Sci. USA 99: 2404-2409.

Fegan, M. and P. Prior (2005) How complex is the Ralstonia solanacearum species complex. In: Allen, C., P. Prior and A.C. Hayward (eds.) Bacterial Wilt Disease and the Ralstonia solanacearum Species Complex, APS Press, Saint Paul, pp. 449 461.

Geethanjali, S., K.-Y. Chen, D.V. Pastrana and J.-F. Wang (2010) Development and characterization of tomato SSR markers from genomic sequences of anchored BAC clones on chromosome 6 . Euphytica 173: 85-97.

Han, Y.-K., J.-S. Min, J.-H. Park, K.S. Han, D.-H. Kim, J.-S. Lee and H.-H. Kim (2009) Screening of tomato cultivars resistant to bacterial wilts. Res. Plant Dis. 15: 198-201.

Hayward, A.C. (1991) Biology and epidemiology of bacterial wilt caused by Pseudomonas Solanacearum. Annu. Rev. Phytopathol. 29: $65-87$.

Huet, G. (2014) Breeding for resistances to Ralstonia solanacearum. Front. Plant Sci. 5: 715 .

Ishihara, T., I. Mitsuhara, H. Takahashi and K. Nakaho (2012) Transcriptome analysis of quantitative resistance-specific response upon Ralstonia solanacearum infection in tomato. PLoS ONE 7: e46763.

Jeong, Y., J. Kim, Y. Kang, S. Lee and I. Hwang (2007) Genetic diversity and distribution of Korean isolates of Ralstonia solanacearum. Plant Dis. 91: 1277-1287.

Jung, E.J., H.J. Joo, S.Y. Choi, S.Y. Lee, Y.H. Jung, M.H. Lee, H.G. Kong and S.-W. Lee (2014) Resistance evaluation of tomato germplasm against bacterial wilt by Ralstonia solanacearum. Res. Plant Dis. 20: 253-258.

Kelman, A. (1954) The relationship of pathogenicity of Pseudomonas solanacearum to colony appearance in a tetrazolium medium. Phytopathology 44: 693-695.

Kim, B., I.S. Hwang, H.J. Lee, J.M. Lee, E. Seo, D. Choi and C.S. Oh (2018) Identification of a molecular marker tightly linked to bacterial wilt resistance in tomato by genome-wide SNP analysis. Theor. Appl. Genet. 131: 1017-1030.

Kim, S.-M. (2018) Identification of novel recessive gene $x a 44(t)$ conferring resistance to bacterial blight races in rice by QTL linkage analysis using an SNP chip. Theor. Appl. Genet. 131: 2733-2743.

Lebeau, A., M.C. Daunay, A. Frary, A. Palloix, J.F. Wang, J. Dintinger, F. Chiroleu, E. Wicker and P. Prior (2011) Bacterial wilt resistance in tomato, pepper, and eggplant: genetic resources respond to diverse strains in the Ralstonia solanacearum species complex. Phytopathology 101: 154-165.

Lee, H.-J., E.-J. Jo, N.-H. Kim, Y. Chae and S.-W. Lee (2011) Disease responses of tomato pure lines against Ralstonia solanacearum strains from Korea and susceptibility at high temperature. Res. Plant Dis. 17: 326-333.

Lin, T., G. Zhu, J. Zhang, X. Xu, Q. Yu, Z. Zheng, Z. Zhang, Y. Lun, S. Li, X. Wang et al. (2014) Genomic analyses provide insights into the history of tomato breeding. Nat. Genet. 46: 1220-1226.

Lopes, C.A. and M. Rossato (2018) History and status of selected hosts of the Ralstonia solanacearum species complex causing bacterial wilt in Brazil. Front. Microbiol. 9: 1228.

Lowe-Power, T.M., C.G. Hendrich, E. von Roepenack-Lahaye, B. Li, D. Wu, R. Mitra, B.L. Dalsing, P. Ricca, J. Naidoo, D. Cook et al. (2018) Metabolomics of tomato xylem sap during bacterial wilt reveals Ralstonia solanacearum produces abundant putrescine, a metabolite that accelerates wilt disease. Environ. Microbiol. 20: 1330-1349.

Mangin, B., P. Thoquet, J. Olivier and N.H. Grimsley (1999) Temporal and multiple quantitative trait loci analyses of resistance to bacterial wilt in tomato permit the resolution of linked loci. Genetics 151: 1165-1172.

McHale, L., X. Tan, P. Koehl and R.W. Michelmore (2006) Plant NBS-LRR proteins: adaptable guards. Genome Biol. 7: 212.

Morel, A., N. Peeters, F. Vailleau, P. Barberis, G. Jiang, R. Berthomé and A. Guidot (2018) Plant pathogenicity phenotyping of Ralstonia solanacearum strains. In: Medina, C. and F.J. LópezBaena (eds.) Host-Pathogen Interactions: Methods in Molecular Biology, Springer, New York.

Murray, M.G. and W.F. Thompson (1980) Rapid isolation of high molecular weight plant DNA. Nucleic Acids Res. 8: 4321-4325.

Niewohner, J., F. Salamini and C. Gebhardt (1995) Development of PCR assays diagnostic for RFLP marker alleles closely linked to alleles Grol and $H 1$, conferring resistance to the root cyst nematode Globodera rostochiensis in potato. Mol. Breed. 1: 65-78.

Pascual, L., E. Albert, C. Sauvage, J. Duangjit, J.-P. Bouchet, F. Bitton, N. Desplat, D. Brunel, M.-C. Le Paslier, N. Ranc et al. (2016) Dissecting quantitative trait variation in the resequencing era: complementarity of bi-parental, multi-parental and association panels. Plant Sci. 242: 120-130.

Prior, P., F. Ailloud, B.L. Dalsing, B. Remenant, B. Sanchez and C. Allen (2016) Genomic and proteomic evidence supporting the division of the plant pathogen Ralstonia solanacearum into three species. BMC Genomics 17: 90.

Salgon, S., M. Raynal, S. Lebon, J.M. Baptiste, M.C. Daunay, J. Dintinger and C. Jourda (2018) Genotyping by sequencing highlights a polygenic resistance to Ralstonia pseudosolanacearum in eggplant (Solanum melongena L.). Int. J. Mol. Sci. 19: 357.

Tomato Genome Consortium (2012) The tomato genome sequence provides insights into fleshy fruit evolution. Nature 485: 635-641.

Schell, M.A. (2000) Control of virulence and pathogenicity genes of Ralstonia Solanacearum by an elaborate sensory network. Annu. Rev. Phytopathol. 38: 263-292.

Scott, J.W., J.F. Wang and P.M. Hanson (2005) Breeding tomatoes for resistance to bacterial wilt, a global view. Acta Hortic. 695: 161172.

The 100 Tomato Genome Sequencing Consortium, S. Aflitos, E. Schijlen, H. de Jong, D. de Ridder, S. Smit, R. Finkers, J. Wang, G. Zhang, N. Li et al. (2014) Exploring genetic variation in the tomato (Solanum section Lycopersicon) clade by whole-genome sequencing. Plant J. 80: 136-148.

Thoquet, P., J. Olivier, C. Sperisen, P. Rogowsky, L. Henri and N. Grimsley (1996a) Quantitative trait loci determining resistance to bacterial wilt in tomato cultivar Hawaii7996. Mol. Plant Microbe Interact. 9: 826-836.

Thoquet, P., J. Olivier, C. Sperisen, P. Rogowsky, P. Prior, G. Anais and N. Grimsley (1996b) Polygenic resistance of tomato plants to bacterial wilt in the French West Indies. Mol. Plant Microbe Interact. 9: 837-842.

Tran, N.H. and B.-S. Kim (2010) Influence of temperature, pathogen strain, inoculum density, seedling age, inoculation method and varietal resistance on infection of pepper seedlings by Ralstonia solanacearum. Hortic. Environ. Biotechnol. 51: 95-100.

Vikal, Y. and D. Bhatia (2017) Genetics and genomics of bacterial blight resistance in rice. In: Li, J.Q. (ed.) Advances in International Rice Research, Intechopen, pp. 175-213.

Wang, J.-F., P. Hanson and J.A. Barnes (1998) Worldwide evaluation 
of an international set of resistance sources to bacterial wilt in tomato. In: Prior, P., C. Allen and J. Elphinstone (eds.) Bacterial Wilt Disease: Molecular and Ecological Aspects, Springer Berlin Heidelberg, Berlin, Heidelberg. pp. 269-275.

Wang, J.-F., J. Olivier, P. Thoquet, B. Mangin, L. Sauviac and N.H. Grimsley (2000) Resistance of tomato line Hawaii7996 to Ralstonia solanacearum Pss4 in Taiwan is controlled mainly by a major strain-specific locus. Mol. Plant Microbe Interact. 13: 6-13.

Wang, J.-F., F.-I. Ho, H.T.H. Truong, S.-M. Huang, C.H. Balatero, V. Dittapongpitch and N. Hidayati (2013) Identification of major QTLs associated with stable resistance of tomato cultivar 'Hawaii 7996' to Ralstonia solanacearum. Euphytica 190: 241-252.

Wang, Y., Y. Zhang, Z. Gao and W. Yang (2018) Breeding for resistance to tomato bacterial diseases in China: challenges and prospects. Hortic. Plant J. 4: 193-207.
Yang, H., J. Jian, X. Li, D. Renshaw, J. Clements, M.W. Sweetingham, C. Tan and C. Li (2015) Application of whole genome re-sequencing data in the development of diagnostic DNA markers tightly linked to a disease-resistance locus for markerassisted selection in lupin (Lupinus angustifolius). BMC Genomics 16: 660 .

Yuliar, Y.A. Nion and K. Toyota (2015) Recent trends in control methods for bacterial wilt diseases caused by Ralstonia solanacearum. Microbes Environ. 30: 1-11.

Zeng, H., L. Xu, A. Singh, H. Wang, L. Du and B.W. Poovaiah (2015) Involvement of calmodulin and calmodulin-like proteins in plant responses to abiotic stresses. Front. Plant Sci. 6: 600.

Zhao, Y., W. Liu, Y.-P. Xu, J.-Y. Cao, J. Braam and X.-Z. Cai (2013) Genome-wide identification and functional analyses of calmodulin genes in Solanaceous species. BMC Plant Biol. 13: 70. 